\title{
Improving articles reporting nutritional studies of extracts in the British Journal of Nutrition and in the Journal of Nutritional Science
}

Investigation of novel nutrients present in the extracts of plants and other organisms that have benefits to health is a rapidly expanding area of research. For example, the number of papers published on the health effects of plant-derived extracts has increased from less than thirty-five per year before 2007 to over eighty per year in 2011 and 2012 (www.webofknow ledge.com search terms: plant extract; nutrition; health), with over 940 primary articles published in total. Robust claims for health benefits from these substances require supporting evidence based on empirical investigations carried out to the highest standards of scientific rigour. Unfortunately, some papers published on such extracts fall short of these standards and so may damage the development and reputation of the field.

The British Journal of Nutrition and the Journal of Nutritional Science aim to publish high-quality nutritional research including nutritional studies on extracts that have potential health benefits. The Directions to Authors for these journals have been revised following an editorial by Calder ${ }^{(1)}$ and after internal consultation in order to set standards of experimentation for nutritional studies of extracts. These revisions are detailed in the Directions to Authors published in June 2013 (see links from http://www. nutritionsociety.org/publications/nutrition-society-journals). They address three specific areas related to experimental design: (1) the characterisation of the extract; (2) the amount of the extract used; (3) the chemical composition of the extract.

The following principles will be applied to manuscripts that report nutritional studies of the effects of extracts on healthrelated outcomes. Failure to comply with these directions will result in the automatic rejection of the manuscript. Studies in which the extract or test compound is described as a medicine or is used in non-physiological quantities will also be rejected. The journals will consider for publication reports of studies involving extracts, provided that the preparation and composition of the extract are defined and controlled. This is to ensure that other researchers can replicate the work. The material from which the extract is prepared must be traceable, subject to quality control and available to other researchers from the source of the starting material stated in the article. The material from which the extract is prepared must be identified by the usual taxonomic classification. Extracts from materials produced in limited geographical regions will be considered, provided that these criteria are met, since the range of production may increase in the future. The method of extraction must be described with the amount of detail that is normally expected in any biological study such that it can be repeated precisely by other researchers. Importantly, the nutrient composition of the extract must be characterised in detail, with particular reference to the putative active components. Measures must be undertaken to control the quality of the composition of the extract.

For studies in whole organisms, the amount of the extract used must fall within a range that could reasonably be expected to be achieved safely in humans, whether the study involves human subjects or animal models of human nutrition, or in animals if they are the specific target for a putative health benefit. Nutritional studies of extracts in in vitro models will be considered for publication, provided that the above criteria are met and that the amount and molecular form of the extract is the same as that which would be encountered by the cell type used in the model if it were in an intact organism.

It is important that authors also consider all of the Directions to Authors when preparing their manuscript.

\section{Graham C. Burdge}

Editor-in-Chief of the British Journal of Nutrition and of the Journal of Nutritional Science, Academic Unit of Human Development and Health, Faculty of Medicine, University of Southampton, Southampton, UK email g.burdge@nutritionsociety.org

\section{Reference}

1. Calder PC (2012) The scope of the British Journal of Nutrition. Br J Nutr 108, 1531. 